\title{
Métodos computacionais para segmentação do disco óptico em imagens de retina: uma revisão
}

\section{Maíla Lima Claro ${ }^{1}$, Rodrigo Veras ${ }^{1}$, Luís Santos ${ }^{1}$, Marcos Frazão $^{1}$, Antonio Carvalho Filho ${ }^{2}$ and Daniel Leite ${ }^{1}$}

\author{
${ }^{1}$ Universidade Federal do Piauí - Teresina and ${ }^{2}$ Universidade Federal do Piauí - Picos \\ *claromaila@gmail.com; rveras@ufpi.edu.br;luisteixeiira@gmail.com; frazaomarcos1@gmail.com; \\ antoniooseas@ufpi.edu.br; oftalmodaniel@gmail.com
}

Submetido: 27/11/2017. Revisado: 05/05/2018. Aceito: 05/06/2018.

\begin{abstract}
Resumo
A utilização de técnicas de processamento digital de imagens (PDI) é destaque no cenário médico para o diagnóstico automático de patologias. Na área oftalmológica o glaucoma é a segunda principal causa da perda de visão no mundo e não possui cura. Atualmente, existem tratamentos para prevenir a perda da visão, contudo a doença deve ser descoberta nos estágios iniciais. O objetivo principal deste artigo é revisar as metodologias e técnicas de segmentação dos limites do disco óptico e escavação. Essas regiões são utilizadas para o cálculo de métricas para classificação do glaucoma e auxílio aos profissionais da área. Os trabalhos mais recentes publicados na área foram classificados em cinco grupos de acordo com a principal técnica de PDI aplicada: agrupamento, superpixel, contorno ativo, morfologia matemática e redes neurais convolucionais. Além disso, foi realizado um levantamento das principais bases de imagens e métricas de avaliação utilizadas.
\end{abstract}

Palavras-Chave: Algoritmos de Agrupamento; Contorno Ativo; Glaucoma; Morfologia matemática; Redes neurais convolucionais; Superpixel.

\begin{abstract}
The use of digital image processing techniques (DIP) is highlighted in the medical scenario for automatic diagnosis of pathologies. In the ophthalmologic area, glaucoma is the second leading cause of vision loss in the world and has no cure. Currently, treatments are used to prevent vision loss, but the disease must be discovered in the early stages. This paper aims to review the methodologies and techniques of optic disc and cup limits segmentation. These regions are used to calculate metrics for classification of glaucoma and assistance to professionals in the area. The most recent published studies were classified into five groups according to the central DIP technique applied: clustering, superpixel, active contour, mathematical morphology and Convolutional Neural Network. Also, a survey was conducted of the main images databases and evaluation metrics used.
\end{abstract}

Key words: Clustering Algorithms; Active Contour; Glaucoma; Mathematical Morphology; Convolutional Neural Network; Superpixel.

\section{Introdução}

O processo de globalização contribuiu significantemente para avanços no campo da ciểncia, principalmente, na área tecnológica. Recentes avanços na aquisição de imagens e no poder computacional dos dispositivos tornaram possível utilizar informações presentes nas imagens médicas. Esses fatos ocasionaram um considerável interesse no desenvolvimento de sistemas automáticos de diagnóstico médico. Esses sistemas são chamados de sistemas de Diagnóstico Auxiliado por Computador 
(CAD - Computer Aided Diagnosis).

Estudos sobre sistemas CAD mostram que eles podem auxiliar os médicos em diversas tarefas, como: medir estruturas anatômicas, monitorar as mudanças pela comparação de imagens sequenciais, diagnosticar e planejar o tratamento. Eles também aliviam o trabalho repetitivo, prevenindo erros por fadiga e aumentando a eficiência no trabalho. As aplicações de CAD mais estabelecidas em áreas médicas envolvem o uso de sistemas automatizados em mamografia, tomografia computadorizada de tórax e ressonância magnética (Doi; 2005).

No campo oftalmológico, esses avanços tornaram possível a prevenção e o tratamento de doenças que há pouco tempo eram consideradas incuráveis. A prevenção primária e a detecção precoce, bem como o acesso a terapêuticas cirúrgicas oftalmológicas, são determinantes para a redução da morbidade ${ }^{1}$ das doenças da visão (Mookiah et al.; 2013).

Foi desenvolvido um grande número de sistemas CAD para o diagnóstico de vários tipos de doenças oculares, como o glaucoma, retinopatia diabética e edema ocular (Mookiah et al.; 2013). Esses sistemas têm potencial para fornecer uma solução alternativa aos programas de triagem em massa, que precisam examinar um grande número de imagens de fundo de olho, o mais rápido possível.

Até agora, a maioria dos sistemas CAD oftalmológicos estão em fase de investigação e não podem ser colocados em uso prático nas clínicas, devido a várias razões. Uma delas é a diversidade de imagens. Em particular, imagens de retina podem ter qualidades muito diferentes devido a vários tipos de lesões e artefatos. Como resultado, é muito difícil projetar um algoritmo de processamento de imagens que seja capaz de lidar com uma grande quantidade de imagens de retina com características heterogêneas (Anitha et al.; 2009).

Segundo Zhang et al. (2014) vários dispositivos de imageamento médico foram desenvolvidos para capturar diferentes partes do olho. Essas modalidades de imageamento são baseadas em várias tecnologias e as imagens capturadas são utilizadas para observar vários sinais de patologia.De acordo com o levantamento feito pelos autores, fotografia digital da retina é a modalidade de imageamento que mais vem atraindo o interesse de pesquisadores. Os autores apontam também que o glaucoma é a doença com o maior número de pesquisas publicadas nas últimas duas décadas.

Existem na literatura vários algoritmos para o processamento e análise de imagens da retina, no entanto, diferentes grupos de pesquisadores tendem a usar diferentes métricas e bancos de imagens para comparar o desempenho desses algoritmos, tornando assim, a comparação entre os métodos uma tarefa complicada e menos fidedigna. Além disso, apesar de utilizar as mesmas medidas de avaliação, diferentes implementações das métricas podem influenciar os resultados finais. O objetivo deste trabalho é apresentar uma revisão e comparação dos principais métodos computacionais, abordados

\footnotetext{
${ }^{1}$ Variável característica das comunidades de seres vivos e referese ao conjunto de indivíduos, dentro da mesma população, que adquirem doenças (ou uma doença específica) num dado intervalo de tempo.
}

na literatura, para a segmentação do nervo óptico nas imagens da retina. A fim de beneficiar o entendimento, foi realizada uma divisão dos métodos em cinco grupos, classificados de acordo com o procedimento relevante empregado: agrupamento, superpixel, contorno ativo, morfologia matemática e redes neurais convolucionais (CNNs - Convolutional Neural Network).

O presente artigo está organizado em sete seções. As Seções 2 e 3 apresentam os conceitos relacionados ao olho humano e ao glaucoma. Nas Seções 4 e 5 são expostas as principais bases de imagens utilizadas e as métricas de segmentação do disco óptico (DO) e escavação para análise de desempenho dos algoritmos. Nas Seções 6 e 7 são apresentados os métodos de segmentação destas regiões para o auxílio ao diagnóstico do glaucoma e por fim, são realizadas discussões sobre os métodos.

\section{Olho humano}

O olho é o órgão responsável pela visão. A sua complexa estrutura aliada à capacidade de interpretação visual do cérebro, permite-nos interagir com o meio ambiente de forma natural. O olho humano é muitas vezes comparado a uma câmera fotográfica, já que ambos usam lentes para focalizar a luz incidente. Enquanto a câmera utiliza o sensor óptico para registrar a imagem, o olho utiliza uma camada especializada de células chamada retina. Porém, as similaridades não se identificam quando se trata da capacidade do olho em focar uma grande variedade de objetos com diferentes tamanhos, luminosidades e contrastes em alta velocidade.

O globo ocular possui um formato aproximadamente esférico com um diâmetro em torno de $25 \mathrm{~mm}$, e é envolvido por três camadas: a camada externa (ou protetora), formada pela córnea e pela esclera; a camada intermediária (ou vascular), compreendendo a íris, a coróide e o corpo ciliar; e a camada interna (ou nervosa), formada pela retina. A estrutura do olho humano é mostrada na Figura 1, a qual corresponde a uma seção longitudinal do globo ocular.

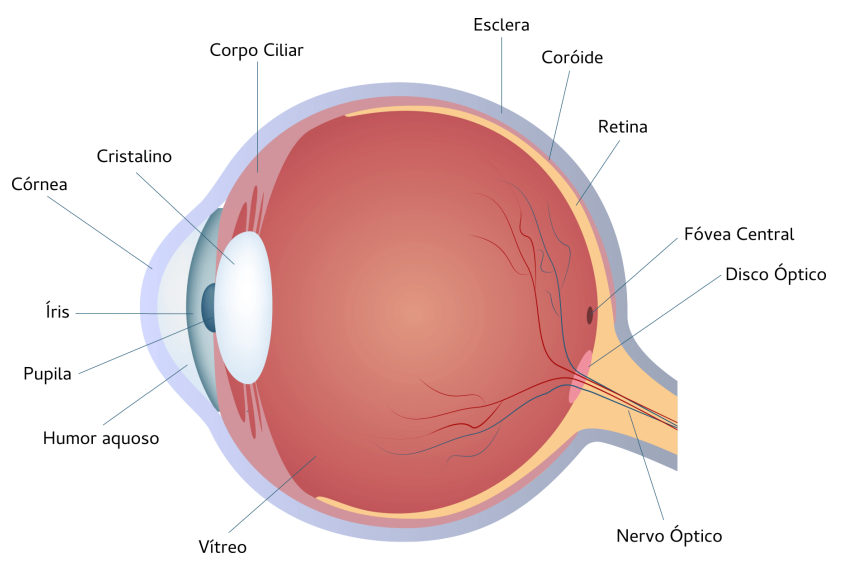

Figura 1: Esquema de um corte longitudinal do olho humano. Adaptado de da Silva Junior and Sasson (1989). 
A retina constitui a camada mais interna do olho, situando-se na sua parede posterior. Quando o olho focaliza uma cena, a imagem correspondente é projetada sobre essa região. A análise da retina pode prover informações sobre mudanças patológicas causadas por doenças oculares locais e sinais recentes de doenças sistêmicas, como: hipertensão, arteriosclerose e diabetes mellitus (Veras et al.; 2013). A Figura 2 apresenta as principais estruturas presentes em uma retina saudável, a saber, o disco óptico, rede de vasos, mácula e fóvea.



Figura 2: Principais estruturas de uma retina saudável.

O DO é a porção do nervo óptico vista no fundo dos olhos, formado pelo encontro de todos os axônios das células ganglionares da retina assim que penetram no nervo óptico. O DO é uma estrutura circular, que apresenta uma tonalidade rosa pálido ou esbranquiçada, tem as bordas bem definidas e está situado no lado nasal da retina e seu diâmetro físico médio é normalmente $1,5 \mathrm{~mm}$ (Nicolela and Vianna; 2016).

O DO não possui células sensíveis à luz. Dessa forma, corresponde ao ponto cego do campo de visão humano. Normalmente, a existência deste ponto cego não é perceptível ao ser humano, pois o cérebro interpreta a informação de cada olho e cobre o ponto cego com informação do outro olho.

\section{Glaucoma}

O glaucoma é uma doença assintomática, neurodegenerativa, crônica e irreversível em que o nervo óptico é progressivamente lesado. Ela é considerada a segunda principal causa de cegueira no mundo, com cerca de 60 milhões de casos em 2010. Uma estimativa sugere que, em 2020, cerca de 80 milhões de pessoas no mundo terão essa doença (Quigley and Broman; 2006).
Essa doença é ocasionada pelo alargamento anormal da escavação (do inglês CUP), parte mais interna, se comparado ao alargamento do DO, e são identificadas usando a relação do raio da escavação e do DO, denominado Cup-to-Disc Ratio (CDR). Um elevado valor do CDR indica a presença de glaucoma no paciente. A Figura 3 mostra as marcações do contorno do DO e da escavação feitas por um oftalmologista. É possível visualizar a diferença das marcações do contorno do DO e da escavação em um olho saudável (Figura 3(a)) e com glaucoma (Figura 3(b)).
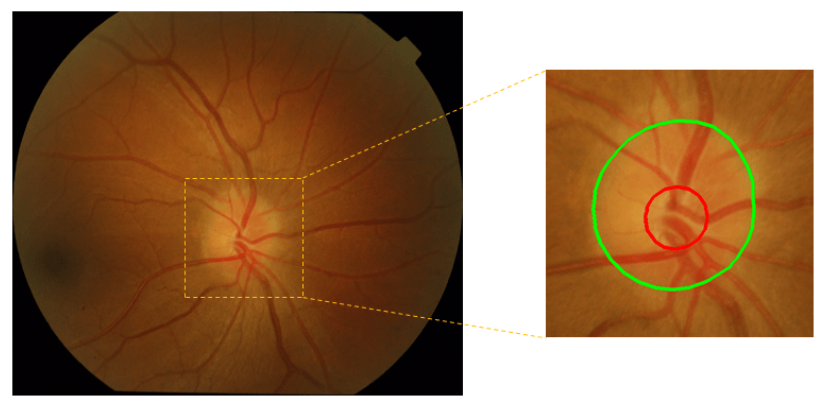

(a) Exemplo de retina saudável.


(b) Exemplo de retina com glaucoma.

Figura 3: Exemplos de marcação do contorno do DO (verde) e Escavação (vermelho) feita por um especialista.

As três principais técnicas de diagnóstico do glaucoma são: 1) avaliação do aumento da pressão intraocular (PIO), 2) avaliação do campo visual e 3) avaliação do nervo óptico. A PIO é medida através de um aparelho chamado tonômetro, que não é sensível o suficiente para ser uma ferramenta de triagem eficaz, porque o glaucoma pode estar presente com ou sem aumento da PIO (Cheng et al.; 2013).

A avaliação do campo visual é feita através da perda de visão, que requer equipamentos especiais presentes nos hospitais. A análise do nervo óptico é mais sensível e superior à medição da PIO ou teste do campo visual para detecção do glaucoma. Essa avaliação é feita por um profissional treinado, de forma manual, subjetiva, demorada e cara (Cheng et al.; 2013). Dessa maneira, o auxílio ao diagnóstico do glaucoma, utilizando imagens digitais da retina, vem sendo amplamente implementado e testado por vários centros de pesquisas, pois aumenta o percentual de acertos, bem como auxilia os profissionais da área em casos mais complexos da doença. 


\section{Bases de Imagens}

A maioria das metodologias de segmentação do disco óptico e da escavação que serão apresentadas nesta pesquisa são testadas em conjuntos de dados disponíveis publicamente. A seguir, é apresentada uma breve descrição das principais bases de imagens utilizadas para avaliar sistemas automáticos de segmentação do disco óptico.

Standard Diabetic Retinopathy Database Calibration Level o - DIARETDBo foi criada com base na justificativa em um excessivo potencial para novos produtos na indústria médica e possíveis reduções nos custos referentes aos cuidados com a saúde (Kauppi et al.; 2006). A base contém 130 imagens de retina com tamanho de $1500 \times 1152$ pixels, sendo 20 saudáveis e 110 contendo sinais de retinopatia diabética. As imagens abrangem uma quantidade desconhecida de ruídos, de aberrações ópticas (dispersão, cromática transversal e lateral, esférica, curvatura de campo, astigmatismo, distorção) e precisão desconhecida da informação fotométrica (cor ou intensidade).

Méthodes d'Evaluation de Systèmes de Segmentation et d'Indexation Dédiées à l'Ophtalmologie Rétinienne MESSIDOR é um projeto financiado pelo Ministério Francês da Pesquisa e Defesa dentro do 2004 TECHNO-VISION, programa para implementação e teste de algoritmos de segmentação. Contém 1200 imagens coloridas do fundo do olho adquirido pelo Hôpital Lariboisière Paris, Faculté de Médecine St. Etienne e LaTIM-CHU de Brest (França). Destas imagens, 800 foram capturadas com dilatação da pupila e 400 sem dilatação. As imagens são $1440 \times 960,2240 \times 1488$ ou $2304 \times 1536$ pixels em tamanho e 8 bits por plano de cor e são fornecidas em formato TIFF. Esta base possui 540 imagens de pacientes não afetados pela Retinopatia Diabética e 660 imagens correspondem aos pacientes afetados pela doença (Decencière et al.; 2014).

Digital Retinal Images for Optic Nerve Segmentation Database - DRIONS-DB é composta pela colaboração conjunta de três organizações espanholas, i.e. Universidad Complutense, Hospital Miguel Servet e Universidad Nacional de Educación a Distancia. Tem 110 imagens retinianas, cada uma com resolução de $600 \times 400$ pixels e o disco óptico segmentado por dois especialistas. As imagens foram adquiridas com uma câmera analógica e centrada aproximadamente no disco óptico. Para obter as imagens em formato digital, elas foram digitalizadas usando um scanner HP-PhotoSmart-S20 de alta resolução, formato Red, Green, Blue - RGB, resolução $600 \times 400$ e 8 bits/pixel. Os contornos independentes de dois especialistas médicos foram coletados usando a ferramenta de software fornecida para a anotação da imagem (Carmona et al.; 2008).

An Open Retinal Image Database for Optic NerveEvaluation - RIM-ONE r1 é uma base de imagens registrada com o intuito de auxiliar os trabalhos de pesquisa que são focados na análise de imagens de retina para localizar, avaliar e detectar o DO (Fumero et al.; 2011). A base é composta de 169 imagens de retina registradas em 3 diferentes regiões da Espanha, com tamanhos que variam de $342 \times 316$ à $869 \times 723$ pixels. O conjunto está dividido em 118 saudáveis, 12 com glaucoma inicial, $14 \mathrm{com}$ glaucoma moderado, 14 com glaucoma profundo e 11 com hipertensão ocular. A base foi criada com a colaboração de 4 oftalmologistas e 1 optometrista. Os padrões ouro de cada imagem estão disponíveis.

An Open Retinal Image Database for Optic NerveEvaluation - RIM-ONE r3 é um conjunto de dados que consiste em 159 imagens de retina, sendo 85 saudáveis e 74 glaucomatosas (PenaBetancor et al.; 2015). As imagens com dimensões $2144 \times 1424$ pixels foram obtidas por meio de uma câmera estereoscópica nonmydriatic Wx Kowa. A base foi primeiramente analisada por dois especialistas e a marcação média das regiões do DO e da escavação foram disponibilizadas. Todas as imagens foram utilizadas, pois todas possuem informações sobre seus padrões ouro.

High-Resolution Fundus - HRF é um banco de dados que foi estabelecido por um grupo de pesquisa colaborativo para apoiar estudos comparativos sobre algoritmos de segmentação automática em imagens de fundo de retina (Budai et al.; 2013). Este banco de dados pode ser usado livremente para fins de pesquisa, ele contém no momento 15 imagens de pacientes saudáveis, 15 imagens de pacientes com retinopatia diabética e 15 imagens de pacientes glaucomatosos, todas com tamanho de $3504 \times 2336$ pixels. Imagens binárias de segmentação de padrão ouro dos vasos estão disponíveis para cada imagem.

Retinal image dataset for optic nerve head (ONH) segmentation - DRISHTI-GS é uma base de imagens que foi desenvolvida e distribuída com o intuito de fornecer meios para avaliação de sistemas CADs na detecção do glaucoma (Sivaswamy et al.; 2014). As imagens foram previamente analisadas por quatro especialistas para a segmentação das regiões do DO e da escavação. A base é composta por 101 imagens de retina, todas foram tiradas com centro no DO, com um "campo de visão" de $30^{\circ}$ e dimensões $2896 \times 1944$ pixels em formato PNG. Apenas 50 desse total possuem informações sobre seus padrões ouro, então normalmente apenas elas são utilizadas para testes.

Algumas dessas bases disponibilizam o padrão ouro de segmentação das regiões do DO, que são as marcações feitas pelos especialistas, enquanto outras não. No segundo caso, os autores desses artigos tiveram a contribuição de especialistas. As bases que possuem o padrão ouro são as DRIONS-DB, RIM-ONEr1, RIM-ONE-r3 e DRISHTI-GS. As bases que não possuem padrão ouro são as DIARETDBo, MESSIDOR e HRF.

A Figura 4 demonstra exemplos de imagens das bases de dados DRIONS-DB, RIM-ONE r1 e DRISHTIGS em cada coluna, respectivamente. Nas Figuras 4(a) e 4(e) encontram-se imagens de duas retinas saudáveis e a Figura 4(c) uma imagem com glaucoma profundo. Já as Figuras 4(b) e 4(d) contém as respectivas imagens da segmentação da região do disco óptico (padrão ouro). Diferente das outras bases, a DRISHTI-GS possui as segmentações das regiões do disco óptico (região cinza e branca) e escavação (região branca), que estão sendo representadas pela Figura 4(f). 

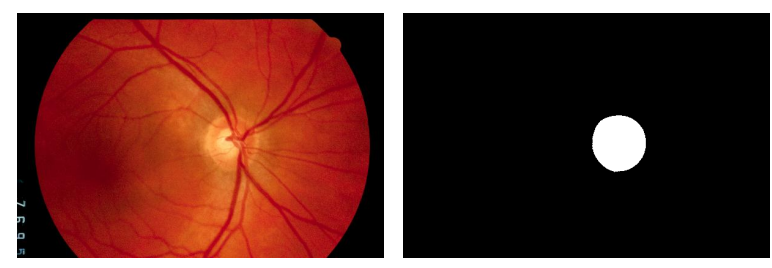

(a) Imagem de retina (b) Padrão Ouro da base saudável. DRIONS-DB.


(c) Imagem de retina com (d) Padrão Ouro da base RIMglaucoma. ONE r1.


(e) Imagem de retina (f) Padrão Ouro da base saudável. DRISHTI-GS.

Figura 4: Exemplos de imagens das bases DRIONS-DB, RIM-ONE r1 e DRISHTI-GS.

\section{Métricas de Avaliação}

O glaucoma envolve uma degeneração progressiva das fibras do nervo óptico, levando a um maior alargamento da escavação (parte mais interna) se comparado ao alargamento do DO (parte mais externa). A representação dessas regiões pode ser visualizada na Figura 5(c), na qual a região do DO (representada por toda a área dentro da marcação verde), e a região da escavação (toda a área dentro da marcação vermelha) estão em evidência. A região que faz parte do $\mathrm{DO}$, mas não faz parte da escavação, é denominada Rima Neural (Neuro Retinal Rim). Todas essas regiões são utilizadas para o cálculo de diferentes métricas de diagnóstico do glaucoma.

A razão escavação/DO (CDR) é uma das métricas de avaliação e é definida como uma relação que compara o diâmetro da escavação com o diâmetro total do DO, indicando a presença do glaucoma caso tenha um elevado valor. Como pode ser observado nas Figuras 5(a) e 5(b), existem dois tipos de CDR que utilizam o diâmetro para o cálculo, sendo eles o vertical e horizontal. A Figura 5(c) está representando a razão da área da escavação/DO (Cup-to-Disc Area Ratio - CDAR), na qual esta relação é definida pela área da escavação sobre a área do DO.

A regra Inferior, Superior, Nasal e Temporal (ISNT) é outra medida de avaliação que afirma que um DO saudável tem uma configuração característica da Rima Neural. A espessura dessa região costuma



(a) CDR Vertical.

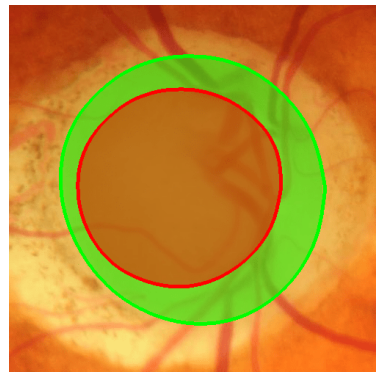

(c) CDR área (CDAR).



(e) Regra da área ISNT.



(b) CDR Horizontal.



(d) Regra ISNT.



(f) Regra DDLS.
Figura 5: Representação das métricas de diagnóstico de glaucoma citadas.

ser maior na região inferior, seguida pelas regiões superior, nasal e temporal (inferior $>$ superior $>$ nasal $>$ temporal). Qualquer alteração nesta ordem pode ser um sinal precoce de lesão (Harizman et al.; 2006), como pode ser observado na Figura $5(\mathrm{~d})$. Outra forma de utilizar o ISNT seria por meio da área, que está representado na Figura 5(e). Nela a área da região inferior tem que ser maior que as outras, seguindo o mesmo princípio da ISNT, para que essa imagem seja considerada saudável.

A Disc Damage Likelihood Scale (DDLS) é uma escala de probabilidade de dano do disco óptico, baseia-se na comparação com o diâmetro da Rima Neural com o diâmetro do DO. Essa métrica de avaliação pode ser observada melhor através da Figura 5(f), onde a menor distância encontrada entre o contorno do DO (marcação verde) e escavação (contorno vermelho) é utilizada sobre o diâmetro do disco óptico.

Para avaliar os resultados de uma segmentação, a maioria dos critérios utilizam uma matriz de confusão, que indica a quantidade de classificações corretas e incorretas para cada uma das classes. A matriz de confusão é criada baseada em quatro valores: Verdadeiro Positivo (VP), são os pixels corretamente informados como "Positivos"; 
Falso Positivo (FP), são os pixels incorretamente informados como "Positivos"; Falso Negativo (FN) refere-se aos pixels incorretamente informados como "Negativos", e Verdadeiro Negativo (VN) que corresponde aos pixels corretamente informados como "Negativos" (Chimieski and Fagundes; 2013).

A partir desses valores, algumas taxas estatísticas podem ser calculadas para avaliar o desempenho dos métodos abordados. A taxas de Sobreposição, Sensibilidade, Especificidade, Acurácia, Precisão e FScore são calculadas por meio das Equações de 1 a 6, respectivamente.

$$
\begin{array}{r}
\text { Sobreposição }=\frac{V P}{V P+F P+F N} \\
\text { Sensibilidade }=\frac{V P}{V P+F N} \\
\text { Especificidade }=\frac{V N}{V N+F P} \\
\text { Acurácia }=\frac{V P+V N}{V P+F P+F N+V N} \\
\text { Precisão }=\frac{V P}{V P+F P} \\
\text { F-Score }=2 * \frac{\text { Precisão } * \text { Sensibilidade }}{\text { Precisão }+ \text { Sensibilidade }}
\end{array}
$$

A taxa de Sobreposição é a interseção da região segmentada com a sua real segmentação, mostrando a proporção de predições corretas para a classe analisada. A Sensibilidade reflete o quanto este é eficaz em identificar corretamente, dentre todos os indivíduos avaliados, aqueles que realmente apresentam a característica de interesse. Já a Especificidade de um método reflete o quanto ele é eficaz em identificar corretamente os indivíduos que não apresentam a condição de interesse. Acurácia é a porcentagem de casos corretamente classificados em um conjunto de teste. Essa taxa mede o quão bem um classificador reconhece instâncias de diversas classes. Precisão reflete a proporção de verdadeiros positivos em relação a todas as predições positivas.

Já para calcular o desempenho de métodos que levam em conta a distância, são calculadas as taxas de Erro Médio Absoluto $\left(E_{m a}\right)$ e o Erro Quadrático Médio $\left(E_{q m}\right)$. Como exemplo, o cálculo do CDR pode ser realizado da seguinte forma: utilizando os resultados obtidos pelos métodos $\left(C D R_{m}\right)$ e o CDR do padrão ouro $\left(C D R_{p o}\right)$ para cada imagem $i$ fornecida pela base, calculamos o Erro Absoluto por imagem $\left(e_{i}\right)$ por meio da Equação 7:

$$
e_{i}=C D R_{p o}-C D m_{i} \text {. }
$$

O $E_{m a}$ e $E_{q m}$ são calculados pelas Equações 8 e 9:

$$
\begin{aligned}
E_{m a} & =\frac{\sum_{i=1}^{n}\left|e_{i}\right|}{n}, \\
E_{q m} & =\frac{\sum_{i=1}^{n} e_{i}^{2}}{n},
\end{aligned}
$$

onde $n$ representa a quantidade de imagens da base.

\section{Métodos de Segmentação}

Foi realizada uma revisão extensiva da literatura da década atual, objetivando identificar quais técnicas estão sendo utilizadas na segmentação do disco óptico e da escavação, para auxílio ao diagnóstico do glaucoma em imagens da retina.

A segmentação do DO e escavação é uma tarefa complexa. Dessa forma, os métodos existentes utilizam diversas técnicas. Dentre os pesquisadores da área não há consenso sobre a melhor forma de classificar esses métodos. Segundo Haleem et al. (2013) os métodos podem ser divididos em duas categorias: métodos baseados em modelo e métodos não baseados em modelo. Já Almazroa et al. (2015), sugere uma classificação baseada na principal técnica de segmentação utilizada. Assim, os autores classificaram os métodos em oito grupos. Avaliamos que a classificação baseada na principal técnica utilizada reflete melhor os trabalhos da última década. Contudo, diferente de Almazroa et al. (2015), classificamos os trabalhos em menos grupos por intender que há interseções entre os grupos propostos por Almazroa et al. (2015). Assim os artigos foram classificados em cinco grupos: algoritmos de Agrupamento, algoritmos de superpixel, Contorno Ativo, Morfologia Matemática e redes neurais convolucionais.

O gráfico apresentado na Figura 6 demonstra a distribuição percentual dos principais trabalhos publicados na última década, onde a técnica de superpixel contempla o maior número de trabalhos encontrados para a segmentação das regiões do disco óptico e escavação para auxílio ao diagnóstico do glaucoma.



Figura 6: Principais técnicas utilizadas para segmentação das regiões do DO e da escavação.

A seguir são apresentados os principais artigos publicados. Ao final de cada subseção são destacadas as vantagens e desvantagens da utilização de cada técnica.

\subsection{Algoritmos de Agrupamento}

A segmentação por meio de agrupamento é realizada pixel a pixel. Usualmente as características utilizadas para representar os pixels são as informações de cor, como os canais RGB e HSV. Os principais algoritmos 
de agrupamentos utilizados na segmentação das regiões do DO são o K-means MacQueen et al. (1967) e o K-means Fuzzy Bezdek et al. (1984).

$\mathrm{O} \mathrm{K}$-means é um método não-supervisionado que divide a imagem em $k$ partes com base em um modelo criado pela média de cada parte. Trabalhos recentes utilizaram o algoritmo com a intenção de segmentar imagens médicas (Ayub et al.; 2016; Vogado et al.; 2016). Uma das desvantagens deste algoritmo é a sua sensibilidade a valores inconsistentes, ruídos e centroides iniciais.

O K-means Fuzzy é um algoritmo nãosupervisionado e tem realizado segmentações de imagens médicas com sucesso (Viswanathan; 2015; Mittapalli and Kande; 2016). Essa técnica é baseada na média de cada grupo e agrupa valores de dados similares utilizando-se de lógica fuzzy para calcular essa semelhança. $O$ algoritmo provou ser útil na produção de resultados eficazes na segmentação de imagens com ruídos, além de utilizar pouca interação humana. Baseando-se nesses algoritmos, várias técnicas para detecção e segmentação do DO utilizando algoritmos de agrupamento têm sido desenvolvidas e aplicadas.

Anusorn et al. (2013) inicialmente removem os vasos por meio do uso de operações morfológicas e do filtro da mediana. A escavação foi segmentada através do uso de limiar no canal de cor verde, do sistema RGB, e usando um método de threshold level-set. Para segmentar o DO foi utilizado o canal de cor vermelho, também do sistema RGB, no algoritmo de Canny Canny (1987) para detecção de bordas, no K-means e no método Variational Level-Set Zhao et al. (1996). Após a segmentação, é desenhada uma elipse em cada região para remodelar as bordas. A base utilizada é privada e foi obtida no Mettapracharak Hospital, em Nakhon Pathom, na Thailândia, contendo 44 imagens (28 saudáveis e 16 com glaucoma). 0 método atingiu, no cálculo do CDR, uma Acurácia de $89 \%$ e um Erro Médio Absoluto de 0,061.

Khalid et al. (2014) aplicaram operações morfológicas para apagar os vasos sanguíneos e suavizar as intensidades ao redor do centro do DO. Posteriormente foi aplicado o K-means Fuzzy no canal de cor verde, do sistema RGB, para segmentar as regiões de escavação e DO. Para analisar a abordagem foi utilizada um base privada de 27 imagens, atingindo uma Acurácia de 90,26\% e $93,70 \%$ na segmentação dos pixels da escavação e DO, respectivamente. Além disso, $88 \%$ dos valores de CDR calculados pela abordagem coincidem aos calculados pelos oftalmologistas.

Ayub et al. (2016) inicialmente fizeram um préprocessamento, no qual foram feitas operações morfológicas para remover os vasos, alargamento de contraste onde uma faixa de níveis de cinza tem seu valor incrementado, enquanto outras faixas de níveis de cinza têm seus valores decrementados, conversão do RGB para o Hue, Saturation, Value - HSV e seguido da passagem de um filtro da mediana no canal V. Posteriormente, foi feita a segmentação representando os pixels utilizando as informações dos sistemas de cor RGB e HSV pré-processados e aplicando o algoritmo $\mathrm{K}$-means. A terceira etapa foi a detecção de bordas das regiões utilizando o algoritmo de Canny, para em seguida realizar o encaixe de uma elipse em cada região. Por fim, foi calculado o CDR.
Eles utilizaram uma base de dados privada coletada no Armed Forces Institute of Ophthalmology (AFIO) em Rawalpindi, Paquistão, contendo 100 imagens (73 normais e 27 glaucomatosas). O método obteve uma Acurácia de $92 \%$ na classificação das imagens e 0,023 de Erro Quadrático Médio no cálculo do CDR.

Mittapalli and Kande (2016) apresentaram um método que segmenta a escavação com um agrupador baseado em um algoritmo de limiarização e o DO com o uso de Contorno Ativo. Os vasos foram detectados a partir de um filtro Gaussian Hermite Kernel Kuo and Woźniakowski (2012), combinados e repintados utilizando a mediana das intensidades dos pixels vizinhos, que não são pertencentes aos vasos, e seguido do filtro da mediana. Foi usada a combinação de ponderação espacial, K-means Fuzzy e técnicas de operações morfológicas, para segmentar a escavação a partir do sistema de cor RGB. Já na segmentação do DO, foi utilizado o canal de verde, do sistema de cor RGB, e aplicado à um modelo de Contorno Ativo, baseado na região implícita encontrada a partir dos pontos de interesse detectados. O sistema proposto foi avaliado em 59 imagens de retina (17 normais e 42 com glaucoma), sendo que 40 são fornecidas por um hospital oftalmológico local e as outras 19 obtidas aleatoriamente de duas bases públicas. O método obteve um F-score de $97,5 \%$ e $89 \%$ na segmentação da escavação e do DO, respectivamente, e um Erro Estimado de 0,13 no cálculo do CDR, 0,12 no cálculo do HCDR (Horizontal Cup-to-Disc Ratio) e 0,15 no CDAR (Cup-to-Disc Area Ratio).

Thakur and Juneja (2017) segmentaram a escavação e o DO utilizando os canais verde e vermelho, respectivamente, do sistema de cor RGB. As segmentações das regiões foram realizadas utilizando operação morfológica de fechamento, um algoritmo de agrupamento intuicionista fuzzy $K$-means, detecção de bordas com o algoritmo de Canny e, por fim, transformada de Hough Duda and Hart (1972). A abordagem foi utilizada em 50 imagens da base de dados DRISHTI-GS e atingiu uma Acurácia de 91,9\% e 94,46\% na segmentação da escavação e DO, respectivamente. Além disso, o método obteve $90 \%$ de concordância dos valores de CDR com o valor apresentado pelo especialista.

Estas técnicas de agrupamento possuem as vantagens de simplicidade na implementação e necessidade de pouco tempo computacional em sua execução. Como desvantagens, apresentam problemas na definição do melhor conjunto de atributos, sensibilidade à ruídos, inicialização dos centroides e definição de qual grupo representa cada região. Para diminuir a influência desses problemas estão surgindo abordagens semissupervisionadas, que utilizam informações prévias dos dados adquiridas com o auxílio de especialistas.

Santos et al. (2017) aplicaram um algoritmo de agrupamento semissupervisionado baseado no K-means Fuzzy para segmentar a escavação e o DO. Os autores representaram os pixels utilizando informações de cor dos sistemas RGB, HSV, HSI, Luv, Lab, CMYK, NTSC YCbCr e CIE XYZ. Posteriormente, usaram técnicas de operações morfológicas e contorno ativo para melhorar os resultados da segmentação, para finalmente fazer o cálculo do CDR. Nos testes foram utilizadas 50 imagens da base de dados DRISHTI-GS e 159 imagens da base RIM- 
Tabela 1: Resumo dos métodos que utilizam Agrupamento. O método marcado com * é semiautomático e os demais são automáticos.

\begin{tabular}{|c|c|c|c|c|}
\hline Autores & Métrica de Avaliação & Base de Imagens & Número de Imagens & Resultado da Avaliação \\
\hline Anusorn et al. (2013) & CDR & Mettapracharak Hospital & 44 & $\begin{array}{l}\text { Acurácia CDR: } 89,00 \% \\
E_{m a} \text { CDR: } 0,061\end{array}$ \\
\hline Khalid et al. (2014) & CDR & Privada & 27 & $\begin{array}{c}\text { Acurácia CDR: } 88,00 \% \\
\text { Acurácia Escavação: 90,26\% } \\
\text { Acurácia DO: } 90,26 \% \\
\text { F-Score Escavação: } 93,70 \%\end{array}$ \\
\hline Ayub et al. (2016) & CDR & $\begin{array}{l}\text { Armedforces institute of } \\
\text { ophthalmology (AFIO) }\end{array}$ & 100 & $\begin{array}{c}E_{m q} \text { CDR: 0,023 } \\
\text { Classificação Glaucoma: } 92,00 \%\end{array}$ \\
\hline Mittapalli and Kande (2016) & $\begin{array}{c}\text { HCDR, VCDR, } \\
\text { CDAR, ISNT e DDLS }\end{array}$ & $\begin{array}{l}\text { Hospital oftalmológico local, } \\
\text { DIARETDBo e RIM-ONE r1 }\end{array}$ & 59 & $\begin{array}{c}\text { F-Score Escavação: } 97,50 \% \\
\text { F-Score DO: } 89,00 \%\end{array}$ \\
\hline Thakur and Juneja (2017) & CDR & DRISHTI-GS & 50 & $\begin{array}{c}\text { Acurácia CDR: 90,00\% } \\
\text { Acurácia Escavação: 91,90\% } \\
\text { Acurácia DO: } 94,46 \%\end{array}$ \\
\hline Santos et al. (2017)* & CDR & DRISHTI-GS e RIM-ONE r3 & 209 & $\begin{array}{c}E_{m a} \text { CDR: 0,0707 } \\
E_{m q} \text { CDR: 0,0095 } \\
\text { Acurácia Escavação: } 97,04 \% \\
\text { Acurácia DO: } 92,24 \%\end{array}$ \\
\hline
\end{tabular}

ONE r3, ambas públicas. A abordagem alcançou uma Acurácia de 97,04\% na segmentação da escavação e $92,24 \%$ na região do DO. Eles atingiram também um Erro Médio Absoluto de 0,0707 e um Erro Quadrático Médio de 0,0095 no cálculo do CDR.

A Tabela 1 apresenta um comparativo entre os trabalhos descritos nesta seção. A partir dessa tabela pode-se perceber que todos os autores utilizaram o CDR como métrica de avaliação do glaucoma. Além disso, a abordagem de Mittapalli and Kande (2016) usou outras medidas que, em alguns casos, também foi feita a avaliação da segmentação do método. Contudo, apesar de utilizarem a mesma regra para diagnóstico, não foi empregada uma métrica comum para analisar os resultados.

Somente em Anusorn et al. (2013), Ayub et al. (2016) e Santos et al. (2017) houve concordância ao utilizar a mesma métrica para analisar o cálculo do CDR. Anusorn et al. (2013) e Santos et al. (2017) utilizaram o Erro Médio Absoluto, no qual o primeiro trabalho apresenta um resultado superior. Nos trabalhos de Ayub et al. (2016) e Santos et al. (2017) os autores avaliaram o CDR usando o Erro Quadrático Médio, onde o segundo trabalho se sobressaiu. Já na utilização das bases de imagens praticamente não ocorreu combinação, em cada trabalho foi utilizado um conjunto diferente, exceto nos trabalhos de Santos et al. (2017) e de Thakur and Juneja (2017), onde ambos usaram a base DRISHTI-GS.

\subsection{Superpixel}

O superpixel é uma abordagem de segmentação de imagens baseada em região, que consiste em particionar a imagem em vários agrupamentos de pixels Ren and Malik (2003). Esta técnica pode ser usada como uma pré-segmentação para redução da quantidade de pixels da imagem ou da segmentação final. A grande importância da técnica de superpixel está associada à redução do custo computacional que estas conferem, em relação às várias tarefas de análise de imagens. Ela particiona a imagem em vários agrupamentos de pixels, fazendo com que as imagens sejam analisadas por regiões.

Cheng et al. (2013) propuseram uma segmentação da região de escavação e disco óptico utilizando a classificação de superpixel. Com a aquisição de imagens de retina, aplica-se o algoritmo Simple Linear Iterative Clustering - SLIC Achanta et al. (2012), que realiza uma extração de característica utilizando histograma. Os atributos são extraídos com o cálculo estatístico do centro (Center Surround Statistics - CSS) Klein and Frintrop (2011). Logo após a extração das características cada superpixel é classificado como pertencente a região do disco ou não. O classificador utilizado nesse trabalho foi a Máquinas de Vetores de Suporte - SVM. Os métodos de segmentação propostos foram avaliados em duas bases de dados com um total de 2326 imagens. Os resultados experimentais mostram um erro de sobreposição médio de $9,5 \%$ e $24,1 \%$ na segmentação do disco óptico e da escavação, respectivamente.

Alghmdi et al. (2015) utilizaram o algoritmo de agrupamento de superpixel SLIC. Foram utilizados dois classificadores, o primeiro linear e o segundo não-linear. O classificador linear é utilizado para classificar uma região como sendo do disco ou não, e o classificador não-linear é utilizado para classificar a região como sendo escavação ou não-escavação. Em seguida, foram aplicadas operações morfológicas, na segmentação da região de escavação e disco óptico. Por último, o cálculo do CDR é realizado com base nos resultados obtidos pela segmentação. Os resultados demonstraram sua eficácia com $92 \%$ de Sensibilidade e $88 \%$ de Especificidade.

Indira et al. (2015) realizaram inicialmente um préprocessamento nas imagens, para depois aplicar o algoritmo de geração de superpixel SLIC. Os superpixels são classificados com base na informação de textura. $\mathrm{O}$ algoritmo de agrupamento $\mathrm{K}$-means e o filtro de Gabor Jones and Palmer (1987) são utilizados para delimitar as bordas das regiões. Após essa etapa da segmentação do DO, é realizada uma segmentação da região de escavação. Por fim, a relação CDR é avaliada para a detecção de glaucoma. Neste trabalho o resultado não foi disponibilizado.

Thorat and Raut (2015) realizaram primeiramente um pré-processamento nas imagens e posteriormente aplicaram o algoritmo SLIC. Em seguida, a extração de características para cada superpixel foi feita utilizando equalização de histograma e cálculo estatístico do centro (CSS). A classificação dos superpixels como disco ou não-disco é feita com o classificador SVM. Após 
Tabela 2: Resumo dos métodos que utilizam superpixel.

\begin{tabular}{|c|c|c|c|c|}
\hline Autores & Métrica de Avaliação & Base de Imagens & Número de Imagens & Resultado da Avaliação \\
\hline Cheng et al. (2013) & CDR & $\begin{array}{c}\text { Singapore Malay Eye } \\
\text { Study (SiMES) e Singapore } \\
\text { Chinese Eye Study (SCES) }\end{array}$ & 2326 & Acurácia CDR: $66 \%$ \\
\hline Alghmdi et al. (2015) & CDR e ISNT & $\begin{array}{l}\text { Moorfields Eye Hospital } \\
\text { in London }\end{array}$ & 12000 & $\begin{array}{l}\text { Acurácia Escavação: } 78 \% \\
\text { Acurácia DO: } 94 \%\end{array}$ \\
\hline Indira et al. (2015) & CDR & Privada & - & - \\
\hline Thorat and Raut (2015) & CDR & $\begin{array}{c}\text { Singapore Malay Eye } \\
\text { Study (SiMES) e Singapore } \\
\text { Chinese Eye Study (SCES) }\end{array}$ & 2326 & - \\
\hline Tan et al. (2015) & CDR & ORIGA-light & 650 & $\begin{array}{c}\text { Acurácia CDR: } 85 \% \\
\text { Acurácia Escavação: } 70,12 \%\end{array}$ \\
\hline Karkuzhali and Manimegala (2017) & CDR e ISNT & DRISHT & 101 & $\begin{array}{c}\text { Acurácia Escavação: } 98,42 \% \\
\text { Acurácia DO: } 97,23 \% \\
\text { Acurácia: } 99 \%\end{array}$ \\
\hline
\end{tabular}

esta etapa aplica-se o algoritmo $\mathrm{K}$-means e o filtro de Gabor, que foram utilizados para detectar as bordas da região. Por fim, o CDR é calculado baseado na segmentação da região do disco e da região de escavação. Os experimentos foram realizados em 2.326 imagens, mas não expõem os resultados.

Tan et al. (2015) utilizaram um pré-processamento nas imagens com o objetivo de extrair os vasos sanguíneos para que em seguida seja aplicado uma normalização de contraste nas imagens. Após essa etapa, é realizado uma segmentação com o uso do superpixel em multi-escala, a qual é realizada pelo algoritmo SLIC. Logo após efetua-se a extração de caraterística. Em seguida, é verificado se os vasos sanguíneos presentes na imagem foram removidos na região aplicada com superpixel. Após isso, é realizado um treinamento e teste, pelo classificador SVM, usando cross validation. Neste trabalho foi avaliada uma base de dados com um total de 650 imagens. O método proposto obteve 0,945 na área sob curva, na segmentação da região de escavação, respectivamente.

Karkuzhali and Manimegala (2017) utilizaram uma estratégia similar a de Cheng et al. (2013). Inicialmente a imagem é segmentada pelo algoritmo SLIC e a classificação dos superpixels é realizada com uma SVM a partir do cálculo estatístico do centro (CSS). A diferença encontra-se no pósprocessamento. Neste trabalho os autores executam operações adaptativas de morfologia matemática (Adaptive Mathematical Morphology - AMM). São realizadas operações de fechamento e abertura com limiares adaptativos nas imagens resultantes das bandas R e G. Os autores avaliaram o desempenho do seu método nas 101 imagens da base DRISHTI-GS.

A Tabela 2 apresenta os artigos expostos acima que utilizam superpixel para a segmentação das regiões de interesse nas imagens digitais da retina. Em todos os trabalhos o CDR foi utilizado para indicar a presença de glaucoma, mas apenas em Cheng et al. (2013) e Thorat and Raut (2015) as bases aplicadas coincidiram. Além disso, dentre as taxas calculadas, os autores Karkuzhali and Manimegala (2017) atingiram os maiores valores de Acurácia na segmentação da escavação e do DO, alcançando 98,42\% e 97,23\% respectivamente.

Os autores citados utilizaram o algoritmo Simple Linear Iterative Clustering (SLIC), na geração de superpixel. Uma vantagem sobre aplicação de superpixel é que ele é mais robusto a ruído na imagem. Contudo, a desvantagem do uso deste método se dá pela etapa de pré-processamento, que exige um maior esforço computacional para a construção das unidades e pelo risco de perder dados relativos às bordas da imagem colocando-as dentro de um superpixel.

\subsection{Contorno Ativo}

Modelos de Contorno Ativo são utilizados para detecção de objetos em uma determinada imagem usando técnicas de evolução de curvas. A ideia básica é começar com uma curva inicial e depois deformar essa curva para o limite do objeto que se deseja segmentar. Essa deformação ocorre sob algumas restrições da imagem (Lotankar et al.; 2015).

Joshi et al. (2011) propuseram um método para a segmentação do disco óptico e da escavação que se baseia em um modelo de contorno ativo e em evidências anatômicas, tais como curvas de vasos. O primeiro passo consiste em localizar o disco óptico e utiliza-lo como uma região de interesse. Após isso, os segmentos dos vasos sanguíneos são identificados através de uma técnica baseada em curvatura e uma operação morfológica de fechamento. Em seguida, o detector de borda Canny é utilizado, a fim de se obter pontos de borda. Sobre esses pontos, a transformada de Hough circular é aplicada para identificar o DO. Por fim, um modelo de contorno ativo é utilizado para realizar a segmentação do DO. Na região do DO são detectadas as curvas de vasos, denominadas $r$ bends. Informações de luminosidade e o conjunto de curvas detectadas são utilizadas para a segmentação da escavação. $O$ método proposto foi testado em um conjunto de imagens obtidas de um hospital. Os autores obtiveram um erro médio absoluto do VCDR de 0,11 e para o erro médio do CDAR um valor de 0,14.

Muramatsu et al. (2011) calcularam a relação CDR em imagens digitais de fundo de retina. $O$ método foi avaliado utilizando 80 imagens, incluindo 25 glaucomatosas e 55 imagens não glaucomatosas, obtidos na própria instituição. A região do DO foi segmentada utilizando o método de Contorno Ativo com as informações de brilho e borda. A segmentação da escavação foi realizada utilizando um mapa de profundidade do DO. As CDRs foram medidas e comparadas com aquelas determinadas por uma segmentação manual fornecida por um especialista da área.

Yin et al. (2012) propõem um método para segmentação do disco óptico e da escavação baseado em um modelo de Contorno Ativo e transformada 
Tabela 3: Resumo dos métodos que utilizam Contorno Ativo.

\begin{tabular}{|c|c|c|c|c|}
\hline Autores & Métrica de Avaliação & Base de Imagens & Número de Imagens & Resultado da Avaliação \\
\hline Joshi et al. (2011) & VCDR e CDAR & Privada & 135 & $\begin{array}{l}E_{m a} \text { VCDR: } 0,11 \\
E_{m a} \text { CDAR: } 0,14\end{array}$ \\
\hline Muramatsu et al. (2011) & CDR & Gifu University Hospital & 80 & $\begin{array}{c}E_{m a} \text { CDR: } 0,11 \\
\text { Acurácia Escavação: } 61 \% \\
\text { Acurácia DO: } 88 \%\end{array}$ \\
\hline Yin et al. (2012) & CDR & Privada & 325 & $E_{m a}$ CDR: 0,100 \\
\hline Kusumandari et al. (2015) & CDR e ISNT & $\begin{array}{l}\text { Cicendo Eye Hospital Bandung } \\
\text { e Bandung Eye Center }\end{array}$ & 64 & Acurácia: $84,38 \%$ \\
\hline Lotankar et al. (2015) & $\begin{array}{l}\text { CDR, CDAR, } \\
\text { HCDR e VCDR }\end{array}$ & Kasturba Medical College & 150 & $\begin{array}{l}\text { Sensibilidade: } 86 \% \\
\text { Especificidade: } 84 \%\end{array}$ \\
\hline
\end{tabular}

de Hough. A primeira etapa consiste na detecção do disco óptico, na qual o canal $\mathrm{R}$ (vermelho) do espaço de cores RGB é utilizado. No entanto, em imagens que apresentam baixa variação de intensidade de pixels na região do DO, é realizado um pré-processamento que aumenta o contraste do disco. Em sequência, o operador de detecção de bordas Canny é usado para criar o mapa de bordas da imagem. Com isso, a transformada de Hough é aplicada, a fim de se obter o círculo que mais se assemelha com o disco óptico. O DO é então utilizado como uma região de interesse para a segmentação da escavação. Nesta etapa, realiza-se a eliminação dos vasos sanguíneos, que são detectados por meio de Local Entropy Thresholding. Por fim, um modelo de contorno ativo é aplicado para extrair o contorno da escavação. $O$ banco de imagens privado ORIGA foi utilizado para testar o método. Os autores obtiveram um erro médio absoluto de CDR de 0,100 .

Kusumandari et al. (2015) compararam o desempenho do método de Contorno Ativo com o do método Ellipse Fit. O resultado deste trabalho demonstra que o sistema de GVF Snake Contour Active $\mathrm{Xu}$ and Prince (1998) provê um melhor resultado, tendo ambos um desempenho com alta Precisão para a relação CDR, no qual o resultado foi de $84,38 \%$ para o método GVF Snake Contour Active e $81,25 \%$ para o método de Ellipse Fit.

Lotankar et al. (2015) fizeram a extração de diversas características, tais como o Cup-to-Disc Ratio (CDR), Cup-to-Disc Area Ratio (CDAR), Horizontal CDR (HCDR) e Vertical CDR (VCDR). O DO é segmentado utilizando o geodesic do modelo de Contorno Ativo e a escavação é detectada usando a informação de cor da banda M do modelo de cor CMYK. A avaliação do desempenho da técnica proposta foi realizada em 150 imagens, compreendendo 75 imagens normais e 75 imagens glaucomatosas, utilizando um conjunto de classificadores supervisionados, nomeadamente Naive Bayes, Support Vector Machine (SVM) e $k$-Nearest Neighbor (KNN). A maior Acurácia obtida foi de $99,22 \%$ utilizando o classificador KNN.

A seguir, é apresentada a Tabela 5, que mostra um comparativo entre as técnicas que utilizam Contorno Ativo para segmentação das regiões de interesse.

Podemos observar que o trabalho de Yin et al. (2011) apresenta uma quantidade de imagens superior aos outros trabalhos, alcançando mais que o dobro das outras. Para testar a técnica do modelo de Contorno Ativo na detecção automática do glaucoma, foi feita uma comparação com o modelo de Ellipse Fit, realizado pelo trabalho de Kusumandari et al. (2015), no qual o modelo de Contorno Ativo foi considerado de maior desempenho, obtendo uma Acurácia de 84,38\%. Outros trabalhos que utilizam Contorno Ativo foram explanados, obtendo resultados importantes, como o trabalho de Lotankar et al. (2015) que obteve uma Acurácia de 99,22\%. Além de que, outra importante vantagem deste trabalho é que pode-se segmentar as regiões do DO e da escavação utilizando imagens de baixa qualidade.

Uma das vantagens deste método se aplica na representação da curva, pois ela permite uma mudança na topologia. Assim, os objetos que são divididos em diversas partes podem ser tratados sem o uso de ferramentas adicionais. A desvantagem é que a curva é atraída para a borda mais significativa em uma vizinhança próxima. Logo, qualquer borda entre a curva inicial e o objeto a ser detectado modifica o resultado final, que seria detectar o objeto desejado. Isso faz com que o método seja extremamente sensível à inicialização.

\subsection{Morfologia Matemática}

A Morfologia Matemática (MM) na área de processamento digital de imagens é particularmente adequada para analisar formas em imagens. A dilatação, erosão, abertura, fechamento, entre outras, são exemplos de operações morfológicas utilizadas no melhoramento da imagem.

Khan et al. (2013) segmentam as regiões do DO e da escavação utilizando os canais de cores verde do modelo RGB e o canal $\mathrm{V}$ do modelo de cor HSV, respectivamente. Posteriormente, são utilizadas técnicas de Morfologia Matemática para a detecção mais precisa dessas regiões, pois estas serão utilizadas nas métricas de avaliação CDR e ISNT. Esta técnica foi aplicada em 50 imagens retinianas e a classificação foi feita manualmente, tendo alcançado uma precisão de $94 \%$. Esta técnica também é debatida por Choudhary and Tiwari (2015), contudo, o método obtém uma Precisão média de $94 \%$ a $96 \%$, o que é considerada melhor do que a abordagem anteriormente mencionada em Khan et al. (2013)

Rajaiah and Britto (2014) segmentam a região do DO utilizando a análise do discriminante linear para extração de características na imagem. Na imagem de saída desse discriminante será usada a equalização do histograma para melhoramento da imagem, na qual após as operações morfológicas são usadas para detecção do DO. Para a segmentação da escavação é aplicada a transformada de Watershed Beucher (1979) no canal verde que é extraído da imagem de entrada. A relação CDR é calculada após determinar o limite do disco óptico e da escavação. 
O processo fornece resultados automatizados sem qualquer intervenção do usuário. 0 método proposto é testado em 50 imagens, coletadas em um banco de dados, e encontrou com sucesso as amostras doentes em 48 casos com a taxa de sucesso de $96 \%$.

Jose and Balakrishnan (2015) utilizam a segmentação das regiões do disco óptico e da escavação na sua metodologia. A região do DO é segmentada usando operações morfológicas e uma metodologia híbrida. Já na segmentação da região da escavação é necessária um pré-processamento da imagem, em que são detectados os vasos sanguíneos e logo após é utilizado o classificador SVM para a remoção dos mesmos. Neste trabalho um valor de CDR superior a 0,5 indica a presença de glaucoma. Verifica-se que o método proposto produz um erro médio de 0,021 (CDR) quando comparado com a observação pericial.

Chauhan and Gulati (2016) apresentam um algoritmo que calcula a relação CDR obtida por meio do processamento de imagens de retina obtidas a partir de Stratus OCT do Sudhalkar Eye Hospital, Vadodara. O processamento de imagem foi realizado em 120 imagens retinianas, sendo 79 olhos glaucomatosos e 41 olhos normais. O método de Otsu e o algoritmo de Watershed foram utilizados para a segmentação de imagens. Operações morfológicas foram utilizadas para a segmentação das regiões da escavação e do disco óptico. A Precisão do algoritmo é da ordem de $94 \%$.

Septiarini et al. (2017) propuseram um método de localização de DO e escavação para uma avaliação de glaucoma assistida por computador. É estabelecido um limiar com base no canal verde da imagem em RGB, que é usado como referência para prever a área inicial do disco. Posteriormente, uma dissimulação de borda é realizada por limiarização com base no espaço de cor vermelha, seguido de detecção de borda. Um método robusto para a segmentação da escavação também é proposto, para a detecção da borda também é feita uma dissimulação da escavação, realizada por limiarização, só que utilizando o canal verde do modelo de cor RGB. São utilizados dois conjuntos de bases de imagens D-I e D-II com 60 e 38 imagens, respectivamente. Os resultados para a localização do disco atinge um $F$-Score médio de 0,96 para $D$-I e 0,96 para D-II. O método de segmentação da escavação atinge um F-Score médio de 0,88 para $\mathrm{D}-\mathrm{I}$ e 0,85 para D-II. Nesta pesquisa é estimado o valor CDR e os valores de ISNT, melhorando assim, a precisão geral do método. Em geral, o resultado obtido neste trabalho é uma evidência de que o método é promissor para a avaliação do glaucoma.

A Tabela 4 apresenta os artigos expostos acima sobre a utilização de Morfologia Matemática para a segmentação das regiões de interesse nas imagens digitais da retina. Podemos observar que os trabalhos de Khan et al. (2013) e Choudhary and Tiwari (2015) utilizaram as mesmas métricas de desempenho e a mesma metodologia para obtenção dos resultados. Contudo, as bases de dados e a quantidade de imagens utilizadas para testes por cada trabalho são distintas.

Choudhary and Tiwari (2015) obtiveram excelentes resultados na detecção do glaucoma utilizando Morfologia Matemática na segmentação das regiões do DO e da escavação, obtendo um valor na ordem de $96 \%$ de acerto nas relações de CDR e ISNT. O trabalho de Khan et al. (2013) também utiliza a mesma técnica do artigo anteriormente falado, contudo apresenta algumas desvantagens em relação a este, na qual a classificação das imagens é feita manualmente e a porcentagem atingida é inferior a do trabalho de Choudhary and Tiwari (2015).

A vantagem da Morfologia Matemática é a sua simplicidade de implementação, que possui três pilares básicos: o elemento estruturante e as operações de erosão e dilatação. A dificuldade desta técnica reside na escolha do elemento estruturante correto para transformar a intuição intelectual em aplicação prática. Ou seja, é a escolha automática de um valor correto do elemento estruturante, sem a necessidade desse valor ser baseado apenas na intuição.

\subsection{Rede Neural Convolucional}

Uma Rede Neural Convolucional é uma classe de rede neural artificial, que vem sendo aplicada com sucesso no processamento e análise de imagens digitais. Uma CNN tende a demandar um nível mínimo de pré-processamento quando comparada a outros algoritmos de classificação de imagens. Esse tipo de rede é usada principalmente em reconhecimento de imagens e processamento de vídeo, embora já tenha sido aplicada com sucesso em experimentos envolvendo processamento de voz e linguagem natural.

Lim et al. (2015) descreve uma solução baseada na aplicação de redes neurais convolucionais para segmentação do DO e da escavação. O disco óptico sem a obstrução dos vasos sanguíneos é evidenciado, assim como, a região da escavação. Os mapas de probabilidade produzidos passam então por um procedimento de refinamento robusto que leva em conta o conhecimento prévio sobre as estruturas da retina. A análise desses mapas de probabilidade permite obter uma estimativa de confiança sobre a exatidão da segmentação, que pode ser usada para direcionar os casos mais desafiadores para inspeção manual. Neste trabalho é utilizado duas bases de dados MESSIDOR E SEED-DB, onde o erro médio absoluto do CDR foi de 0,0608.

Priyanka et al. (2017)propôs uma segmentação automática de estruturas anatômicas em imagens de fundo de olho, como vaso sanguíneo e disco óptico, usando CNN. O método atingiu 95,64\% na Acurácia da classificação de vasos sanguíneos. Além disso, a escavação e o disco óptico são segmentados utilizando o Fuzzy C-means. A estratégia foi testada em uma base de imagem privada, com os valores de CDR obtidos sendo comparados aos valores da base.

Sevastopolsky (2017) realizou uma segmentação automática das regiões do DO e da escavação utilizando a rede neural convolucional U-Net. Para a segmentação destas duas regiões é utilizado Contrast Limited Adaptive Histogram Equalization (CLAHE) Szeliski (2010) como pré-processamento das imagens. Esse método equaliza o contraste da imagem, alterando assim, a cor das regiões e interpolando o resultado entre elas. Para a segmentação da escavação a imagem é primeiro cortada por uma delimitação do DO (com margem de cada lado), que é adquirida a partir do algoritmo de segmentação do DO. Este 
Tabela 4: Resumo dos métodos que utilizam Morfologia Matemática.

\begin{tabular}{|c|c|c|c|c|}
\hline Autores & Métrica de Avaliação & Base de Imagens & Número de Imagens & Resultado da Avaliação \\
\hline Khan et al. (2013) & CDR e ISNT & $\begin{array}{c}\text { Hamilton Eye Institute } \\
\text { (HEI-MED), Friedrich-Alexander } \\
\text { University of Erlangen- } \\
\text { Nuremberg e Messidor }\end{array}$ & 50 & Acurácia: $94 \%$ \\
\hline Rajaiah and Britto (2014) & CDR & Privada & 50 & Acurácia: $96 \%$ \\
\hline Jose and Balakrishnan (2015) & CDR & $\begin{array}{l}\text { Little Flower Hospital } \\
\text { \& Research Centre }\end{array}$ & - & $E_{m a}$ CDR: 0,021 \\
\hline Choudhary and Tiwari (2015) & CDR e ISNT & Hrf, Drions e optic-disc.org & 90 & Acurácia: $96,7 \%$ \\
\hline Chauhan and Gulati (2016) & CDR & Sudhalkar Eye Hospital & 120 & Acurácia: $94 \%$ \\
\hline Septiarini et al. (2017) & CDR e ISNT & Privada & 98 & $\begin{array}{c}\text { F-Score Escavação: } 86 \% \\
\text { F-Score DO: } 96 \%\end{array}$ \\
\hline
\end{tabular}

Tabela 5: Resumo dos métodos que utilizam Redes Neurais Convolucionais.

\begin{tabular}{|c|c|c|c|c|}
\hline Autores & Métrica de Avaliação & Base de Imagens & Número de Imagens & Resultado da Avaliação \\
\hline Lim et al. (2015) & CDR & MESSIDOR e SEDD-DB & - & $E_{m a}$ CDR: 0,0608 \\
\hline Priyanka et al. (2017) & CDR e Acurácia & Privada & - & Acurácia vasos sanguíneos: $95,64 \%$ \\
\hline Sevastopolsky (2017) & $\begin{array}{c}\text { F-score e } \\
\text { sobreposição }\end{array}$ & $\begin{array}{l}\text { DRIONS-DB, DRISHTI-GS } \\
\text { e RIM-ONE v.3 }\end{array}$ & 319 & $\begin{array}{c}\text { F-Score Escavação: } 83,5 \% \\
\text { F-Score DO: } 94,5 \% \\
\text { Sobreposição Escavação: } 72 \% \\
\text { Sobreposição DO: } 89 \%\end{array}$ \\
\hline
\end{tabular}

trabalho obteve um $F$-score da segmentação da região do DO no valor de $89 \%$ nas duas bases de imagens utilizadas a RIM-ONE v.3 e a DRIONS-DB. O valor da sobreposição foi de em média 94,5\% nas duas bases. A região da escavação variou de uma base para outra, tendo obtido um $F$-score de $85 \%$ na base de dados DRISHTI-GS e $82 \%$ na base RIM-ONE v.3, tendo uma média de $83,5 \%$. O valor da sobreposição foi de $75 \%$ na base de dados DRISHTI-GS e $69 \%$ na base RIMONE v.3, obtendo uma média de $72 \%$.

As CNNs vem inovando as tarefas de visão computacional nos últimos anos e atualmente oferecem soluções de última geração em segmentação, classificação de imagens e outras tarefas de reconhecimento de imagens. Outra vantagem das redes neurais convolucionais como principais ferramentas de aprendizagem profunda é sua expansão, pois a mesma rede pode geralmente reconhecer vários padrões em diferentes imagens de diferentes objetos.

\section{Conclusões}

Neste trabalho fornecemos uma revisão abrangente dos algoritmos utilizados para a detecção e segmentação do DO e da escavação. Essas regiões são as principais estruturas da cabeça do nervo óptico e são utilizadas para diagnosticar o glaucoma. Existem muitos desafios na estrutura do nervo óptico que acabam limitando uma segmentação mais precisa das regiões do DO e da escavação, como os vasos sanguíneos que dificultam a segmentação precisa dos limites da escavação. Por isso a maioria dos trabalhos apresentados utilizam alguma técnica de detecção e remoção desses vasos.

Os trabalhos expostos nesta revisão diferem quanto aos conjuntos de dados das imagens. Algumas abordagens utilizaram um pequeno conjunto de dados, enquanto outras utilizaram grandes conjuntos de dados para o treinamento e teste dos algoritmos. A maioria dos métodos foram testados usando uma quantidade desbalanceada entre imagens glaucomatosas e saudáveis. Em suma, para um melhor entendimento e comparação das segmentações das regiões do DO e da escavação, foi realizada uma divisão, baseada em cinco métodos, sendo eles: algoritmos de agrupamento, algoritmos de superpixel, contorno ativo, morfologia matemática e redes neurais convolucionais.

Foram apresentadas as bases de dados mais comumente utilizadas para o rastreio automático do glaucoma e as métricas de avaliação que os trabalhos expostos utilizaram. Além disso, foi feita uma listagem das relações CDR, ISNT, dentre outras, utilizadas para diagnosticar o glaucoma. Dessa maneira, o objetivo principal foi apresentar algumas das metodologias atuais de detecção e segmentação e dar ao leitor uma visão geral da pesquisa existente.

Em termos gerais. Conclui-se que ainda há necessidade de avanços na área. Um dos desafios no processamento de imagens de glaucoma é a habilidade de diagnosticar a doença em bases de dados com características distintas. Dentre os trabalhos referenciados poucos realizaram testes em mais de uma base de dados. Dessa forma, os métodos atuais não são viáveis para uso em programas de triagem. Dentre as técnicas utilizadas, o uso de redes neurais convolucionais vem ganhando espaço e demonstrando ser uma ferramenta poderosa. Contudo, será necessário a utilização de um grande conjunto de imagens para o treinamento dessas redes.

\section{Agradecimentos}

Este artigo teve o apoio da Fundação de Apoio à Pesquisa do Estado do Piauí (FAPEPI).

\section{Referências}

Achanta, R., Shaji, A., Smith, K., Lucchi, A., Fua, P. and Süsstrunk, S. (2012). Slic superpixels compared to state-of-the-art superpixel methods, IEEE transactions on pattern analysis and machine intelligence 34(11): 2274-2282.

Alghmdi, H., Tang, H. L., Hansen, M., O'Shea, A., 
Al Turk, L. and Peto, T. (2015). Measurement of optical cup-to-disc ratio in fundus images for glaucoma screening, International Workshop on Computational Intelligence for Multimedia Understanding, IEEE, pp. 1-5.

Almazroa, A., Burman, R., Raahemifar, K. and Lakshminarayanan, V. (2015). Optic disc and optic cup segmentation methodologies for glaucoma image detection: A survey, Journal of Ophthalmology 2015: 1-28.

Anitha, J., Vijila, C. K. S. and Hemanth, D. J. (2009). An overview of computational intelligence techniques for retinal disease identification applications, International Journal of Reviews in Computing 5: 29-46.

Anusorn, C. B., Kongprawechnon, W., Kondo, T., Sintuwong, S. and Tungpimolrut, K. (2013). Image processing techniques for glaucoma detection using the cup-to-disc ratio, Thammasat International Journal of Science and Technology 18(1): 22-34.

Ayub, J., Ahmad, J., Muhammad, J., Aziz, L., Ayub, S., Akram, U. and Basit, I. (2016). Glaucoma detection through optic disc and cup segmentation using $\mathrm{k}$-mean clustering, International Conference on Computing, Electronic and Electrical Engineering, IEEE, pp. 143-147.

Beucher, S. (1979). Use of watersheds in contour detection, Proceedings of the International Workshop on Image Processing, CCETT.

Bezdek, J. C., Ehrlich, R. and Full, W. (1984). Fcm: The fuzzy c-means clustering algorithm, Computers \& Geosciences 10(2-3): 191-203.

Budai, A., Bock, R., Maier, A., Hornegger, J. and Michelson, G. (2013). Robust vessel segmentation in fundus images, International Journal of Biomedical Imaging .

Canny, J. (1987). A computational approach to edge detection, Readings in Computer Vision, Elsevier, pp. 184-203.

Carmona, E. J., Rincón, M., García-Feijoó, J. and Martínez-de-la Casa, J. M. (2008). Identification of the optic nerve head with genetic algorithms, Artificial Intelligence in Medicine 43(3): 243-259.

Chauhan, K. and Gulati, R. (2016). Diagnosis of glaucoma using cup to disc ratio in stratus oct retinal images, International Conference on Information and Communication Technology for Intelligent Systems, Vol. 1, Springer, pp. 507-516.

Cheng, J., Liu, J., Xu, Y., Yin, F., Wong, D. W. K., Tan, N.-M., Tao, D., Cheng, C.-Y., Aung, T. and Wong, T. Y. (2013). Superpixel classification based optic disc and optic cup segmentation for glaucoma screening, Transactions on Medical Imaging 32(6): 1019-1032.

Chimieski, B. F. and Fagundes, R. D. R. (2013). Association and classification data mining algorithms comparison over medical datasets, Journal of Health Informatics 5(2).
Choudhary, K. and Tiwari, S. (2015). Ann glaucoma detection using cup-to-disk ratio and neuroretinal rim, International Journal of Computer Applications 111(11): 8-14.

da Silva Junior, C. and Sasson, S. (1989). Biologia, Atual.

Decencière, E., Zhang, X., Cazuguel, G., Laÿ, B., Cochener, B., Trone, C., Gain, P., Ordonez, R., Massin, P., Erginay, A. et al. (2014). Feedback on a publicly distributed image database: the messidor database, Image Analysis \& Stereology 33(3): 231-234.

Doi, K. (2005). Current status and future potential of computer-aided diagnosis in medical imaging, The British Journal of Radiology 78(suppl_1): s3-s19.

Duda, R. O. and Hart, P. E. (1972). Use of the hough transformation to detect lines and curves in pictures, Communications of the ACM 15(1): 11-15.

Fumero, F., Alayón, S., Sanchez, J., Sigut, J. and Gonzalez-Hernandez, M. (2011). Rim-one: An open retinal image database for optic nerve evaluation, International Symposium on Computer-Based Medical Systems, IEEE, pp. 1-6.

Haleem, M. S., Han, L., Hemert, J. and Lia, B. (2013). Automatic extraction of retinal features from colour retinal images for glaucoma diagnosis: A review, Computerized Medical Imaging and Graphics 37(8): 581-596.

Harizman, N., Oliveira, C., Chiang, A., Tello, C., Marmor, M., Ritch, R. and Liebmann, J. M. (2006). The isnt rule and differentiation of normal from glaucomatous eyes, Archives of Ophthalmology 124(11): 1579-1583.

Indira, K., Vinodhini, J. and Sneha, J. (2015). Optic disc and optic cup segmentation for glaucoma screening adopting superpixel classification, International Journal of Pharmacy e Technology 7(1): 8390-8400.

Jones, J. P. and Palmer, L. A. (1987). An evaluation of the two-dimensional gabor filter model of simple receptive fields in cat striate cortex, Journal of neurophysiology 58(6): 1233-1258.

Jose, A. M. and Balakrishnan, A. A. (2015). A novel method for glaucoma detection using optic disc and cup segmentation in digital retinal fundus images, International Conference on Circuit, Power and Computing Technologies, IEEE, pp. 1-5.

Joshi, G. D., Sivaswamy, J. and Krishnadas, S. (2011). Optic disk and cup segmentation from monocular color retinal images for glaucoma assessment, Transactions on Medical Imaging 30(6): 1192-1205.

Karkuzhali, S. and Manimegala, D. (2017). Computational intelligence-based decision support system for glaucoma detection, Biomedical Research 18(11).

Kauppi, T., Kalesnykiene, V., Kamarainen, J.-K., Lensu, L., Sorri, I., Uusitalo, H., Kälviäinen, H. and Pietilä, J. (2006). Diaretdbo: Evaluation database and methodology for diabetic retinopathy algorithms, Machine Vision and Pattern Recognition 
Research Group, Lappeenranta University of Technology, Finland 133: 134.

Khalid, N. E. A., Noor, N. M. and Ariff, N. M. (2014). Fuzzy c-means (fcm) for optic cup and disc segmentation with morphological operation, Procedia Computer Science 42: 255-262.

Khan, F., Khan, S. A., Yasin, U. U., ul Haq, I. and Qamar, U. (2013). Detection of glaucoma using retinal fundus images, International Conference on Biomedical Engineering, IEEE, pp. 1-5.

Klein, D. A. and Frintrop, S. (2011). Centersurround divergence of feature statistics for salient object detection, Computer Vision (ICCV), 2011 IEEE International Conference on, IEEE, pp. 2214-2219.

Kuo, F. Y. and Woźniakowski, H. (2012). Gausshermite quadratures for functions from hilbert spaces with gaussian reproducing kernels, BIT Numerical Mathematics 52(2): 425-436.

Kusumandari, D. E., ArisMunandar and Redhyka, G. G. (2015). The comparison of gvf snake active contour method and ellipse fit in optic disc detection for glaucoma diagnosis, International Conference on Automation, Cognitive Science, Optics, Micro Electro-Mechanical System, and Information Technology, IEEE, pp. 123-126.

Lim, G., Cheng, Y., Hsu, W. and Lee, M. L. (2015). Integrated optic disc and cup segmentation with deep learning, Tools with Artificial Intelligence (ICTAI), 2015 IEEE 27th International Conference on, IEEE, pp. 162-169.

Lotankar, M., Noronha, K. and Koti, J. (2015). Detection of optic disc and cup from color retinal images for automated diagnosis of glaucoma, Conference on Electrical, Computer and Electronics, IEEE, pp. 1-6.

MacQueen, J. et al. (1967). Some methods for classification and analysis of multivariate observations, Proceedings of the fifth Berkeley symposium on mathematical statistics and probability, Vol. 1, Oakland, CA, USA, pp. 281-297.

Mittapalli, P. S. and Kande, G. B. (2016). Segmentation of optic disk and optic cup from digital fundus images for the assessment of glaucoma, Biomedical Signal Processing and Control 24: $34-46$.

Mookiah, M. R. K., Acharya, U. R., Chua, C. K., Lim, C. M., Ng, E. and Laude, A. (2013). Computeraided diagnosis of diabetic retinopathy: A review, Computers in Biology and Medicine 43(12): 2136 2155 .

Muramatsu, C., Nakagawa, T., Sawada, A., Hatanaka, Y., Yamamoto, T. and Fujita, H. (2011). Automated determination of cup-to-disc ratio for classification of glaucomatous and normal eyes on stereo retinal fundus images, Journal of Biomedical Optics 16(9): 096009-096009.

Nicolela, M. T. and Vianna, J. R. (2016). Optic nerve: clinical examination, Pearls of Glaucoma Management, Springer, pp. 17-26.
Pena-Betancor, C., Gonzalez-Hernandez, M., Fumero-Batista, F., Sigut, J., Medina-Mesa, E., Alayon, S. and de la Rosa, M. G. (2015). Estimation of the relative amount of hemoglobin in the cup and neuroretinal rim using stereoscopic color fundus images, Investigate Ophthalmology \& Visual Science 56(3): 1562-1568.

Priyanka, R., Shoba, S. G. and Therese, A. B. (2017). Segmentation of optic disc in fundus images using convolutional neural networks for detection of glaucoma, 4(5).

Quigley, H. A. and Broman, A. T. (2006). The number of people with glaucoma worldwide in 2010 and 2020, British Journal of Ophthalmology 90(3): 262267.

Rajaiah, R. P. and Britto, R. J. (2014). Optic disc boundary detection and cup segmentation for prediction of glaucoma, International Journal of Science, Engineering and Technology Research 3(10): 2665-2672.

Ren, X. and Malik, J. (2003). Learning a classification model for segmentation, International Conference on Computing, Electronic and Electrical Engineering, IEEE, p. 10.

Santos, L., Veras, R., Rabelo, R., Aires, K. and Aires, O. (2017). A seeded fuzzy c-means based approach to automatic cup-to-disc ratio measurement, International Conference on Systems, Man, and Cybernetics, IEEE, pp. 1075-1080.

Septiarini, A., Harjoko, A., Pulungan, R. and Ekantini, R. (2017). Optic disc and cup segmentation by automatic thresholding with morphological operation for glaucoma evaluation, Signal, Image and Video Processing 11(5): 945-952.

Sevastopolsky, A. (2017). Optic disc and cup segmentation methods for glaucoma detection with modification of $\mathrm{u}$-net convolutional neural network, Pattern Recognition and Image Analysis 27(3): 618-624.

Sivaswamy, J., Krishnadas, S. R., Joshi, G. D., Jain, M. and Tabish, A. S. U. (2014). Drishtigs: Retinal image dataset for optic nerve head (onh) segmentation, International Symposium on Biomedical Imaging, IEEE, pp. 53-56.

Szeliski, R. (2010). Computer vision: algorithms and applications, Springer Science \& Business Media.

Tan, N.-M., Xu, Y., Goh, W. B. and Liu, J. (2015). Robust multi-scale superpixel classification for optic cup localization, Computerized Medical Imaging and Graphics 40: 182-193.

Thakur, N. and Juneja, M. (2017). Clustering based approach for segmentation of optic cup and optic disc for detection of glaucoma, Current Medical Imaging Reviews 13(1): 99-105.

Thorat, S. G. and Raut, S. (2015). Glaucoma screening based on super pixel classification, International Journal of Engineering and Techniques 1(3): 24-28. 
Veras, R., Medeiros, F., Silva, R. and Ushizima, D. (2013). Assessing the accuracy of macula detection methods in retinal images, International Conference on Digital Signal Processing, IEEE, pp. 1-6.

Viswanathan, P. (2015). Fuzzy c means detection of leukemia based on morphological contour segmentation, Procedia Computer Science 58: 84-90.

Vogado, L. H. S., de M S Veras, R., Andrade, A. R., e Silva, R. R. V., de Araujo, F. H. D. and de Medeiros, F. N. S. (2016). Unsupervised leukemia cells segmentation based on multispace color channels, International Symposium on Multimedia, IEEE, pp. 451-456.

$\mathrm{Xu}, \mathrm{C}$. and Prince, J. L. (1998). Snakes, shapes, and gradient vector flow, IEEE Transactions on image processing 7(3): 359-369.

Yin, F., Liu, J., Ong, S. H., Sun, Y., Wong, D. W., Tan, N. M., Cheung, C., Baskaran, M., Aung, T. and Wong, T. Y. (2011). Model-based optic nerve head segmentation on retinal fundus images, Engineering in Medicine and Biology Society, IEEE, pp. 2626-2629.

Yin, F., Liu, J., Wong, D. W. K., Tan, N. M., Cheung, C., Baskaran, M., Aung, T. and Wong, T. Y. (2012). Automated segmentation of optic disc and optic cup in fundus images for glaucoma diagnosis, International Symposium on Computer-based Medical Systems, IEEE, pp. 1-6.

Zhang, Z., Srivastava, R., Liu, H., Chen, X., Duan, L., Wong, D. W. K., Kwoh, C. K., Wong, T. Y. and Liu, J. (2014). A survey on computer aided diagnosis for ocular diseases, BMC Medical Informatics and Decision Making 14(80).

Zhao, H.-K., Chan, T., Merriman, B. and Osher, S. (1996). A variational level set approach to multiphase motion, Journal of computational physics 127(1): 179-195. 